\title{
Respiratory distress in term newborns: Can we predict the outcome?
}

\author{
*Nimesha Chamidani Gamhewage ${ }^{1}$, Hemali Jayakodi ${ }^{2}$, Janaki Samarakoon ${ }^{3}$, Sajini de Silva ${ }^{3}$, LPC \\ Saman Kumara ${ }^{3}$
}

Sri Lanka Journal of Child Health, 2020; 49(1): 30-34

\begin{abstract}
Introduction: Respiratory distress in term newborns is due to a variety of causes and nearly seven percent of term babies develop respiratory distress during the early neonatal period. Numerous challenges are faced by clinicians working in resource poor settings, when deciding on resource allocation and transfer to other units, as it is difficult to predict the babies who might later need advanced respiratory support.
\end{abstract}

Objectives: To determine the incidence and aetiology of early onset respiratory distress in term babies and to identify predictors of congenital pneumonia, need for ventilation and mortality.

Method: A prospective descriptive cross sectional study was conducted at Castle Street Hospital for Women on 200 term babies developing respiratory distress within the first 2 hours of birth. One minute Apgar score, Silverman score, birth weight and details regarding management were recorded.

Results: Incidence of early onset respiratory distress was $8.2 \%$, the commonest aetiologies being transient tachypnoea of newborn, congenital pneumonia and meconium aspiration syndrome. Silverman score of more than 5 on first assessment, birth weight, presence of risk factors for sepsis and low Apgar score were associated with the need for invasive ventilation, while low Apgar score was a predictor for mortality. High Silverman score and presence of risk factors for early onset neonatal sepsis were associated with congenital pneumonia.

Conclusions: Among term babies born at Castle Street Hospital for Women, the incidence of $\mathrm{RD}$

\footnotetext{
${ }^{1}$ University of Sri Jayewardenepura, Sri Lanka, ${ }^{2}$ Regional Director of Health Services, Anuradhapura, Sri Lanka, ${ }^{3}$ Castle Street Hospital for Women, Colombo, Sri Lanka

*Correspondence: nimeshagamhewage@gmail.com
}

(iD https://orcid.org/0000-0001-5129-4158

(Received on 25 April 2018: Accepted after revision on 21 June 2019)

The authors declare that there are no conflicts of interest

Personal funding was used for the project.

Open Access Article published under the

Commons Attribution CC-BY (c) (i) License within first 2 hours of birth was $8.2 \%$, the common aetiologies being TTN, congenital pneumonia and MAS. While Silverman score more than 5 on first assessment and low first minute Apgar were strongly predictive of the need for invasive ventilation, low first minute Apgar score was a predictor for mortality.

DOI: http://dx.doi.org/10.4038/sljch.v49i1.8895

(Key words: Respiratory distress, newborns, incidence, aetiology, predictors of outcome)

\section{Introduction}

Respiratory distress (RD) is one of the commonest causes for neonatal intensive care unit admission in both preterm and term babies, contributing significantly to neonatal mortality and morbidity ${ }^{1}$. It is estimated to affect approximately seven percent of term newborns during their early neonatal period ${ }^{2}$. Manifestations of RD include tachypnoea, grunting, chest wall recessions, nasal flaring and cyanosis ${ }^{1}$. Various aetiologies such as pathology of lung parenchyma, disorders of airway, congenital heart disease, early onset neonatal sepsis, neurological abnormalities and multiple metabolic derangements could be responsible for this clinical entity ${ }^{1}$.

Transient tachypnoea of the newborn (TTN) was found to be the commonest cause for RD among term babies in a study conducted by Hameed and co-workers, while meconium aspiration syndrome (MAS), congenital heart disease and congenital pneumonia were also found to be significant contributors. In their study population, the percentage of $\mathrm{RD}$ in full term newborns was $2.16 \%{ }^{3}$. Respiratory distress syndrome (RDS), which was often overlooked in the past, is increasingly being recognized as an aetiological factor for RD in term babies as well ${ }^{3},{ }^{4}$.

In a cohort of preterm and term babies with $\mathrm{RD}$, John BM et al recognised gestational age and birth weight as independent predictors of mortality. Meanwhile, gestational age and Apgar score were independent predictors for requirement of mechanical ventilation ${ }^{5}$. Furthermore, in another study, which enrolled both preterm and term babies, Back SY et al identified that among babies with TTN, requirement for mechanical ventilation was associated with lower umbilical artery $\mathrm{pH}$ and lower Apgar score at 1 minute ${ }^{6}$.However, as above mentioned studies included both term as well as 
preterm babies, it is difficult to identify the unique factors affecting the outcome of term babies, who constitute a large proportion of the babies with respiratory distress. In a French population based study, overall incidence of mechanically ventilated term babies with TTN, RDS and MAS were found to be $0.72 \%, 0.38 \%$ and $0.61 \%$, respectively. In term newborns, first minute Apgar score equal to or less than 3, caesarean section, presence of meconium-stained amniotic fluid and abnormal cardiotocography were identified as risk factors for severe respiratory disease ${ }^{7}$.

In a Sri Lankan study, conducted at a tertiary care hospital, all the extreme preterm babies had surfactant deficient lung disease and 93\% had required mechanical ventilation ${ }^{8}$. However, there is a dearth of studies evaluating respiratory distress in term newborns in our country. Therefore, the incidence, prevalence, causative factors and outcome of our babies with respiratory distress is not known. Identification of the causes of respiratory distress is important for provision of basic facilities for neonatology units and in future planning. Furthermore, knowledge of early predictors of outcome enables us to optimize monitoring, treatment and timely transfer of babies to higher centres.

\section{Objectives}

To determine:

1. Incidence of early onset RD in term babies at Castle Street Hospital for Women.

2. Aetiology of early onset RD in term babies

3. Predictors of requirement for invasive ventilation, mortality, and final diagnosis being congenital pneumonia

\section{Method}

A descriptive cross sectional study was conducted at the Castle Street Hospital for Women from October 2018 to January 2019 among term neonates. Those with birthweight less than $1600 \mathrm{~g}$, babies needing intubation at birth and babies with antenatally diagnosed congenital heart disease or diaphragmatic hernia were excluded. RD in the newborn was diagnosed in the presence of at least one of the following criteria: respiratory rate of $60 / \mathrm{min}$ or more, increased respiratory effort (subcostal in-drawing, xiphoid retraction, suprasternal in-drawing), nasal flaring, expiratory grunt and cyanosis at room temperature ${ }^{1}$. The babies were examined and findings were recorded by the Medical Officer on duty and diagnosis was reviewed by two Consultant Neonatologists at Castle Street Hospital for Women and the treatment was carried out according to the unit policy. The babies were monitored for the following outcomes: mortality, need for invasive ventilation and congenital pneumonia. The variables assessed as predictors were: presence of a single or more risk factor for sepsis (maternal fever, prolonged rupture of membranes for more than 18 hours, maternal HSV, previous baby who had Group B Streptococcus infection, chorioamnionitis) ${ }^{9}$, Silverman score on first assessment, first minute Apgar score, and birth weight.

Silverman score is a tool which evaluates five clinical parameters namely, upper chest movement, lower chest retractions, xiphoid retractions, dilatation of nares and expiratory grunting. This tool is used to quantify the level of respiratory distress in newborns ${ }^{10}$. In addition, it does not require additional information, such as blood gas analysis.

The following diagnostic criteria were used to determine the aetiology for RD.

1. TTN: RD with chest $\mathrm{x}$-ray showing good inflation and perihilar streaking and normal blood culture and inflammatory markers ${ }^{11}$.

2. RDS: $\mathrm{RD}$ with chest $\mathrm{x}$-ray showing a reticular granularity appearance with air bronchogram and a normal blood culture ${ }^{11}$.

3. MAS: Baby born through meconium stained amniotic fluid, developing RD and chest $\mathrm{x}$-ray appearance of over-inflation, coarse opacities and flattening of the diaphragm $^{11}$.

4. Congenital pneumonia: Chest x-ray finding of lobar infiltration in the presence of either positive blood culture, elevated C-reactive protein $>10 \mathrm{mg} / \mathrm{dl}$, absolute neutropenia $<2000 / \mathrm{ml}$ or elevated immature granulocyte fraction $>20 \%{ }^{11}$.

5. Spontaneous pneumothorax: Chest $\mathrm{x}$-ray findings consistent with pneumothorax, and which had occurred spontaneously ${ }^{11}$.

6. Idiopathic persistent pulmonary hypertension: $\quad$ specific 2 D echocardiographic findings of right to left shunt, tricuspid regurgitation and ventricular septal deviation towards right ventricle $^{11}$. Babies with secondary pulmonary hypertension (e.g. secondary to MAS, congenital pneumonia) were not included under this category ${ }^{11}$.

7. Congenital heart disease: specific $2 \mathrm{D}$ echocardiographic findings

Sample size was calculated using the formula $\mathrm{N}=$ $10 \mathrm{~K} / \mathrm{P}^{12}$. ( $\mathrm{K}=$ number of covariates, $\mathrm{P}=$ proportion of infants with distress due to congenital pneumonia, which was considered as $25 \%{ }^{13}$ ). Over this period, 200 consecutive term babies, who had 
developed RD within the first 2 hours of birth were enrolled, after obtaining informed written consent.

Statistical analysis was done using SPSS software. Frequencies and percentages were calculated to describe the characteristics of the study group. Three logistic regression models were run for the three stated outcomes: requirement for invasive ventilation, mortality and final diagnosis being congenital pneumonia. Independent variables were; presence of a single or more risk factor for sepsis, Silverman score on first assessment, first minute Apgar score and birth weight.

Ethical clearance was obtained from the Ethics Review Committee of Castle Street Hospital for Women and informed written consent was obtained from all mothers/ guardians of the babies enrolled for the study.

\section{Results}

During the study period 2439 live term babies were born at Castle Street Hospital for Women and 8.2\% of them developed RD within the first 2 hours of birth. Among babies with RD, 103 (51.5\%) were boys, while 97 were girls. Mean birth weight was 2648 g. In 39 babies, RD resolved by two hours of age, and hence these babies were handed over to the mother without starting antibiotics or subjecting them to any investigations. Table 1 describes the causes for early onset RD in our cohort. TTN, congenital pneumonia and MAS were the common contributors. In the study cohort, 193 (96.5\%) babies survived, while seven (3.5\%) babies died.

Table 1: Aetiology of respiratory distress (RD) in first two hours of birth

\begin{tabular}{|l|c|}
\hline \multicolumn{1}{|c|}{ Aetiology } & Number of Babies (\%) \\
\hline RD settled by two hours of age and therefore no investigations were performed & $39(19.5)$ \\
\hline Transient tachypnoea of the newborn & $60(30.0)$ \\
\hline Meconium aspiration syndrome & $29(14.5)$ \\
\hline Congenital pneumonia & $48(24.0)$ \\
\hline Neonatal sepsis without pneumonia & $15(07.5)$ \\
\hline Spontaneous pneumothorax & $01(0.5)$ \\
\hline Idiopathic persistent pulmonary hypertension & $04(2.0)$ \\
\hline Antenatally undiagnosed congenital heart disease & $02(1.0)$ \\
\hline Antenatally undiagnosed diaphragmatic hernia & $02(1.0)$ \\
\hline
\end{tabular}

It is interesting to note that, all the babies who recovered within the first two hours of age remained asymptomatic, and did not need any intervention until discharge. On the other hand, none of the babies with congenital pneumonia, MAS, idiopathic pulmonary hypertension and spontaneous pneumothorax recovered by four hours of age.

In the study group, $141(70.5 \%)$ babies needed only nasal prong oxygen for respiratory support. Out of fifty four babies who received non-invasive ventilation initially, 33 required augmentation of respiratory support to an invasive form within 24 hours of admission. Five babies had received invasive ventilation, without a trial of non-invasive ventilation.

With regard to predictors of outcome (Table 2), Silverman score of more than 5 on first assessment and presence of one or more risk factor for sepsis had a significant association with congenital pneumonia in this group of babies with early onset respiratory distress $(\mathrm{p}<0.01))$. However, first minute Apgar score and birth weight were not significant risk factors $(p>0.05)$. Requirement of invasive ventilation during the first 24 hours of life was associated with a Silverman score of more than 5 on first assessment $(p<0.01)$, low first minute Apgar score of $0-3(p<0.01)$, birth weight $(p<0.01)$ and presence of risk factors for sepsis $(p<0.05)$. On the other hand, the only risk factor associated with mortality was low first minute Apgar score $(\mathrm{p}<0.05$ in first minute Apgar 0-3 and 4-6 groups).

None of the babies with TTN, neonatal sepsis without pneumonia and spontaneous pneumothorax needed invasive ventilation (Table 3 ). 
Table 2: Predictors of outcome

\begin{tabular}{|l|l|c|c|c|c|}
\hline \multirow{5}{*}{ Outcome } & \multicolumn{1}{c|}{ Risk factor } & OR & \multicolumn{2}{c|}{ 95\% CI } & P value \\
\cline { 3 - 6 } & & & Lower & Upper & \\
\hline \multirow{5}{*}{$\begin{array}{l}\text { Congenital } \\
\text { Pneumonia }\end{array}$} & Silverman score > 5 on first assessment & 2.866 & 1.353 & 6.072 & 0.006 \\
\cline { 2 - 6 } & Presence of risk factors for sepsis & 3.819 & 1.846 & 7.899 & 0.000 \\
\cline { 2 - 6 } & First minute Apgar score of 4-6 & .733 & .158 & 3.389 & 0.691 \\
\cline { 2 - 6 } & First minute Apgar score of 0-3 & .844 & .205 & 3.481 & 0.815 \\
\cline { 2 - 6 } & Birth weight (in units of 100 grams) & .879 & .771 & 1.002 & 0.054 \\
\hline \multirow{5}{*}{$\begin{array}{l}\text { Invasive } \\
\text { ventilation }\end{array}$} & Silverman score > 5 on first assessment & 54.918 & 13.468 & 223.93 & 0.000 \\
\cline { 2 - 6 } & Presence of risk factors for sepsis & 3.492 & 1.127 & 10.827 & 0.030 \\
\cline { 2 - 6 } & First minute Apgar score of 4-6 & 1.719 & .342 & 8.638 & 0.511 \\
\cline { 2 - 6 } & First minute Apgar score of 0-3 & 8.201 & 1.684 & 39.947 & 0.009 \\
\cline { 2 - 6 } & Birth weight (in units of 100 grams) & .740 & .595 & .921 & 0.007 \\
\hline \multirow{5}{*}{ Mortality } & Silverman score > 5 on first assessment & 3.078 & 0.189 & 50.168 & 0.430 \\
\cline { 2 - 6 } & Presence of risk factors for sepsis & 2.191 & 0.153 & 31.376 & 0.563 \\
\cline { 2 - 6 } & First minute Apgar score of 4-6 & 38.102 & 1.972 & 736.286 & 0.016 \\
\cline { 2 - 6 } & First minute Apgar score of 0-3 & 177.994 & 8.319 & 3808.334 & 0.001 \\
\cline { 2 - 6 } & Birth weight (in units of 100 grams) & .464 & .240 & .900 & 0.023 \\
\hline
\end{tabular}

Table 3: Requirement for mechanical ventilation, according to the final diagnosis

\begin{tabular}{|l|c|}
\hline \multicolumn{1}{|c|}{ Diagnosis } & Number (\%) of babies needing invasive ventilation \\
\hline Transient tachypnoea of the newborn & $0(0)$ \\
\hline Meconium aspiration syndrome & $15(51.7)$ \\
\hline Congenital pneumonia & $16(33.3)$ \\
\hline Neonatal sepsis without pneumonia & $0(0)$ \\
\hline Spontaneous pneumothorax & $0(0)$ \\
\hline Persistent pulmonary hypertension & $03(75)$ \\
\hline Congenital heart disease & $02(100)$ \\
\hline Diaphragmatic hernia & $02(100)$ \\
\hline
\end{tabular}

\section{Discussion}

Our incidence of RD is higher than regional studies, which describe an incidence varying from 2.1 to $2.8^{3,14}$, but in keeping with world statistics ${ }^{2}$. Our findings suggest that a high Silverman score $(>5$ on first assessment) and low first minute Apgar (0-3) are associated with the need for invasive ventilation. The Silverman score is a convenient, yet objective tool to quantify RD in neonates and hence could be utilized in any setup. In fact, Hedstrome $\mathrm{AB}$ et al has demonstrated that in babies with $\mathrm{RD}$, irrespective of gestational age, a high Silverman score bears a strong association with advancement of the respiratory support provided. Furthermore, they suggested that a score equal to or more than 5 in first hour of life is more likely to require an increase in the respiratory support within the first 24 hours of life ${ }^{10}$, which is in keeping with our results. These observations are of pivotal importance, as they can be used as a guide to decide on allocation of ventilators or transfer to a centre with facilities, while avoiding unnecessary transfers and delayed transfers.

Of note, a low first minute Apgar score determines the mortality, which can be seen in both groups with first minute Apgar scores of 0-3 and 4-6 respectively. However, as the mortality is low, with only seven neonatal deaths due to $\mathrm{RD}$, we could observe huge OR ranges, resulting in poor reliability of the above finding. Therefore, we recommend further studies on this regard with high event rates.

Our study has several strengths. Firstly, the cohort of neonates belongs to mothers of diverse social classes and ethnic groups. In addition, in contrast to many studies, where only those who needed admission to neonatal intensive care units had been evaluated, our study assessed all the babies who presented with $\mathrm{RD}$, irrespective of the requirement for admission. Finally, a large number of babies were recruited for the study, which improves the reliability of results. However, both Apgar score and Silverman score might depend on the medical officer attending the baby, and therefore the major limitation of the study is the possibility of interobserver variability, as it is not possible to allocate a single assessor to assess every baby with RD. Nevertheless, we have taken precautions by displaying the Silverman score at observation room and delivery sites, and training was given to all the Medical Officers to improve the accuracy. 


\section{Conclusions}

Among term babies born at Castle Street Hospital for Women, the incidence of RD within first 2 hours of birth was $8.2 \%$, the common aetiologies being TTN, congenital pneumonia and MAS. While Silverman score more than 5 on first assessment, and low first minute Apgar, were strongly predictive of the need for invasive ventilation, low first minute Apgar score was a predictor for mortality.

\section{Acknowledgements}

We acknowledge Dr Buddhima Jayasinghe (Consultant Neonatologist), all the babies, their parents, doctors and other staff at Castle Street Hospital for Women, for their assistance.

\section{References}

1. Flidel-rimon O, Shinwell E. Respiratory distress in the term and near-term Infant. NeoReviews 2005; 6: e289-e297. https://doi.org/10.1542/neo.6-6-e289

2. Edwards MO, Kotecha SJ, Kotecha S. Respiratory distress of the term newborn infant. Pediatric Respiratory Reviews 2013; 14:29-37.

https://doi.org/10.1016/j.prrv.2012.02.002 PMid: 23347658

3. Hameed NN, Al-janabi MK, Al-reda YI. Respiratory distress in full term newborns. Iraqi Postgradguate Medical Journal 2007; 6(3):233-9.

4. Liu J. Respiratory distress syndrome in full-term neonates. Journal of Neonatal Biology 2012; S1:1-2. https://doi.org/10.4172/2167-0897.S1$\mathrm{e} 001$

5. John B, Venkateshwar V, Dagar V. Predictors of outcome in neonates with respiratory distress. Jourbal of Nepal Paediatric Society 2015; 35(1):31-7. https://doi.org/10.3126/jnps.v35i1.11868

6. Bak SY, Shin YH, Jeon JH, Park KH, Kang JH, Cha DH, et al. Prognostic factors for treatment outcomes in transient tachypnoea of the newborn. Pediatrics International 2012; 54(6): 875-80. https://doi.org/10.1111/j.1442200X.2012. 03693.x

PMid: 22747488
7. Gouyon JB, Ribakovsky C, Ferdynus C, Quantin C, Sagot P. Severe respiratory disorders in term neonates. Paediatric and Perinatal Epidemiology 2008; 22(1): 2230.

8. Gamhewage NC, Weerasekara M, Wijayaratne J. Pre-discharge neonatal morbidity and mortality in extremely preterm babies managed at Sri Jayewardenepura Teaching Hospital. Sri Lanka Journal of Child Health 2018; 47:155-8.

https://doi.org/10.4038/sljch.v47i2.8483

9. Oddie S, Embleton ND. Risk factors for early onset neonatal group B streptococcal sepsis: case-control study. British Medical Journal 2002; 325: 308-11.

https://doi.org/10.1136/bmj.325.7359.308

PMid: 12169506 PMCid: PMC117770

10. Hedstrom AB, Gove NE, Mayock DE, Batra M. Performance of the Silverman Andersen Respiratory Severity Score in predicting $\mathrm{PCO} 2$ and respiratory support in newborns: A prospective cohort study. Journal of Perinatology 2018; 38:505-11 https://doi.org/10.1038/s41372-018-00493

PMid: 29426853 PMCid: PMC5998375

11. Hermansen CL, Mahajan A. Newborn respiratory distress. American Family Physician 2015; 92(11): 994-1002.

12. MedCalc statistical software. Available from:

Shttps://www.medcalc.org/manual/logistic _regression.php

13. Santosh S, Kushal KK, Adarsha E. A clinical study of respiratory distress in newborn and its outcome. Indian Journal of Neonatal Medicine and Research 2013;1(1):2-4.

14. Swarnakumar K, Swarnakumar M. Neonatal respiratory distress in early neonatal period and its outcome. International Journal of Biomedical and Advance Research 2015; 6(09): 643-7. 